Exci t at i on of macr onagnet ohydr odynami $c$ mode due to mul ti scal e i nt er act i on in a quasi - st eady equi I i bri um for red by a bal ance bet ween mi cr ot urbul ence and zonal fl ow

\begin{tabular}{|l|l|}
\hline $\begin{array}{l}\text { j our nal or } \\
\text { publ i cat } i \text { on } \mathrm{titl} \text { e }\end{array}$ & Physi cs of Pl asnas \\
\hline vol une & Vol .14 \\
\hline number & I ssue 4 \\
\hline page r ange & pp. 040702-1 - 040702- 4 \\
\hline year & $2007-04$ 01 \\
\hline URL & ht t p: //hdl . handl e. net /10655/3859 \\
\hline
\end{tabular}




\title{
Excitation of macromagnetohydrodynamic mode due to multiscale interaction in a quasi-steady equilibrium formed by a balance between microturbulence and zonal flow
}

\author{
A. Ishizawa a) and N. Nakajima \\ National Institute for Fusion Science, Toki 509-5292, Japan
}

(Received 22 January 2007; accepted 20 February 2007; published online 16 April 2007)

\begin{abstract}
This is the first numerical simulation demonstrating that a macromagnetohydrodynamic (macro-MHD) mode is excited as a result of multi-scale interaction in a quasi-steady equilibrium formed by a balance between microturbulence and zonal flow based on a reduced two-fluid model. This simulation of a macro-MHD mode, a double tearing mode, is accomplished in a reversed shear equilibrium that includes zonal flow and turbulence due to kinetic ballooning modes. In the quasi-steady equilibrium, a macroscale fluctuation that has the same helicity as the double tearing mode is a part of the turbulence. After a certain period of time, the macro-MHD mode begins to grow. It effectively utilizes free energy of the equilibrium current density gradient and is destabilized by a positive feedback loop between zonal flow suppression and magnetic island growth. Thus, once the macro-MHD appears from the quasi-equilibrium, it continues to grow steadily. This simulation is more comparable with experimental observations of growing macro-MHD activity than earlier MHD simulations starting from linear macroinstabilities in a static equilibrium. (C) 2007 American Institute of Physics. [DOI: 10.1063/1.2716669]
\end{abstract}

Macromagnetohydrodynamic (macro-MHD) activities substantially degrade magnetic confinement of toroidal plasmas by producing global fluctuations, and the evolution of these activities is observed in experiments. ${ }^{1}$ Such macroMHD instabilities have been analyzed by nonlinear MHD simulations starting from linear instability growth under a static equilibrium. ${ }^{2-4}$ However, observations in the experiment apparently include microturbulence and zonal flow, ${ }^{5,6}$ and the macro-MHD can nonlinearly originate from turbulent fluctuations. In fact, MHD activities are observed before the disruption in reversed shear plasmas with a transport barrier related to zonal flows and microturbulence, ${ }^{1}$ and microturbulence is observed in Large Helical Device plasmas that usually exhibit MHD activities. ${ }^{7}$ In these experiments, the turbulence can affect macro-MHD in several ways through multiscale interactions. In order to understand the growth of fluctuation observed in the experiments, we have to carry out nonlinear numerical simulation including not only the MHD instability but also the microturbulence and zonal flow created by the turbulence.

Multiscale interactions described in Fig. 1 play key roles in understanding effects of microturbulence on macro-MHD mode. A typical multiscale interaction in the magnetic confinement is the interaction between microturbulence and zonal flow. ${ }^{5}$ Effects of microturbulence on macro-MHD mode through nonlinear mode coupling are studied theoretically and numerically, and they are described by a negative eddy viscosity or by an anomalous resistivity. ${ }^{8-12}$ On the other hand, the zonal flow caused by the turbulence can also affect the macro-MHD instability through the shearing of its radial structure. These effects of turbulence on macro-MHD should be simultaneously taken into account in numerical

\footnotetext{
${ }^{a)}$ Electronic address: ishizawa@nifs.ac.jp
}

simulations. Our goal is to understand the mechanism of macroscale MHD instability in the reversed shear plasmas based on the analysis of multiscale interactions among macroscale MHD, microturbulence, and zonal flows.

In this letter, we obtain a quasi-steady equilibrium established by a balance between microturbulence and zonal flow and demonstrate that a macroscale MHD mode appears in the quasi-steady equilibrium by numerically solving reduced two-fluid equations. We also present the mechanism of macro-MHD development from turbulent fluctuations. This MHD activity spreads the microturbulence over the plasma because it breaks magnetic surfaces globally.

We carry out three-dimensional numerical simulations of a reduced set of two-fluid equations that extends the standard reduced two-fluid equations, ${ }^{13}$ by including temperature gradient effects. ${ }^{14-16}$ The equations are

$$
\begin{aligned}
& \frac{d n}{d t}=-n_{\mathrm{eq}} \nabla_{\|} v_{e \|}+K\left[n_{\mathrm{eq}} \Phi-p_{e}\right]+\mu_{n} \nabla_{\perp}^{2} n, \\
& n_{\mathrm{eq}} \frac{d v_{\|}}{d t}=-\nabla_{\|} p+\mu_{v} \nabla_{\perp}^{2} v_{\|}, \\
& n_{\mathrm{eq}} \frac{d Q}{d t}=-\nabla_{\|} J-K[p]+\widetilde{a} \nabla_{\perp} \cdot\left[\nabla_{\perp} \Phi, p_{i}\right]+\mu_{Q} \nabla_{\perp}^{2} Q,
\end{aligned}
$$$$
\beta \frac{\partial \psi}{\partial t}=-\nabla_{\|} \Phi+\nabla_{\|} p_{e}+\eta_{L} v_{e \|}+\eta J
$$ 


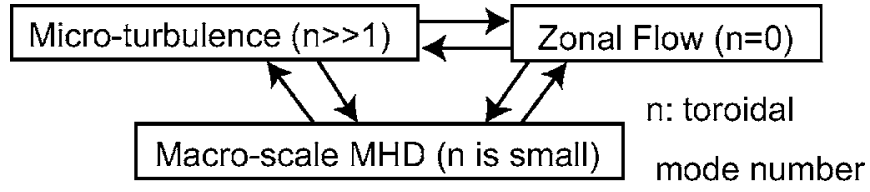

FIG. 1. Schematic drawing of multiscale interactions.

$$
\begin{aligned}
\frac{d T_{i}}{d t}= & -(\Gamma-1)\left(T_{\mathrm{eq}} \nabla_{\|} v_{\|}+\kappa_{L} T_{i}\right)-T_{\mathrm{eq}} K\left[( \Gamma - 1 ) \left(\Phi+T_{i}\right.\right. \\
& \left.\left.+T_{\mathrm{eq}} / n_{\mathrm{eq}} n\right)+\Gamma T_{i}\right]+\mu_{T} \nabla_{\perp}^{2} T_{i},
\end{aligned}
$$

where $d f / d t=\partial f / \partial t+\widetilde{a}[\Phi, f], \quad \nabla_{\|} f=\epsilon \partial f / \partial \zeta-\beta \widetilde{a}[\psi, f], \quad K[f]$ $=2 \epsilon[r \cos \theta, f], J=\nabla_{\perp}^{2} \psi, Q=\nabla_{\perp}^{2} \Phi, \psi=\psi_{\mathrm{eq}}+\tilde{\psi} / \tilde{a}, \Phi=\tilde{\Phi} / \tilde{a}$, $n=n_{\mathrm{eq}}+\tilde{n} / \widetilde{a}, \quad T_{i}=T_{\mathrm{eq}}+\widetilde{T}_{i} / \widetilde{a}, \quad T_{e}=\tau T_{\mathrm{eq}}, \quad p_{i}=n_{\mathrm{eq}} T_{\mathrm{eq}}+T_{\mathrm{eq}} \tilde{n} / \widetilde{a}$ $+n_{\mathrm{eq}} \widetilde{T}_{i} / \widetilde{a}, p_{e}=\tau n_{\mathrm{eq}} T_{\mathrm{eq}}+\tau T_{\mathrm{eq}} \tilde{n} / \widetilde{a}, p=p_{i}+p_{\varrho}, v_{e \|}=v_{\|}+J / n_{\mathrm{eq}}, \widetilde{a}$ $=a / \rho_{i}, \eta_{L}=\sqrt{\pi / 2 \pi m_{e} / m_{i}}\left|\nabla_{\|}\right|, \kappa_{L}=(\Gamma-1) \sqrt{8 T_{\text {eq }} / \tau\left|\nabla_{\|}\right|}$. In these equations, $n, v_{\|}, \Phi, \psi, T_{i}, \rho_{i}, \epsilon=a / R, R$, and $a$ are the electron density, the parallel ion velocity, the electric potential, the flux function, the ion temperature, Larmor radius, the inverse of aspect ratio, the major radius, and the minor radius, respectively. The normalizations are $\left(t v_{\mathrm{ti}} / a, r / \rho_{i}, \rho_{i} \nabla_{\perp}, a \nabla_{\|}, e \Phi / T_{0}, \psi / \beta B_{0} \rho_{i}, n / n_{0}, T / T_{0}, v_{\|} / v_{\mathrm{ti}}\right)$ $\rightarrow\left(t, r, \nabla_{\perp}, \nabla_{\|}, \Phi, \psi, n, T, v_{\|}\right)$. In the numerical calculations we employ 128 toroidal modes and 256 poloidal modes that distribute within $1 \leqslant m / n \leqslant q(r / a=0.93)$, and 256 uniform grid points in the radial direction, where $m$ and $n$ are poloidal and toroidal mode numbers, respectively. We set $\epsilon=0.25$, $\rho_{i} / a=1 / 80, \tau=1$, the artificial dissipations $\mu_{Q}=\mu_{n}=\mu_{v}=\mu_{T}$ $=m^{4} \times 10^{-7}$, and the normalized resistivity $\eta=4 \times 10^{-4}$, which corresponds to $S=1.6 \times 10^{6}$. With respect to boundary conditions, the plasma is assumed to be surrounded by a perfectly conducting wall. Numerical accuracy was checked by comparing the case of 256 radial grid points with the case of 400 radial grid points and by confirming that energy spectra for toroidal and poloidal mode numbers sufficiently decrease in high wave number regions.

We consider a reversed shear tokamak plasma with $\beta$ $=1 \%$ and weak collisions. The equilibrium $q$-profile, density profile, and temperature profile are $q=1.05+2(r / a)^{2}$ $+1 /(3 r / a+0.01)^{4}, \quad n_{\mathrm{eq}}=0.8+0.2 \exp \left[-2(r / a)^{2}\right], \quad$ and $T_{\mathrm{eq}}$ $=0.55+0.45\left[1-(r / a)^{2}\right]^{2}$, respectively. This equilibrium is unstable against the $(m, n)=(2,1)$ double tearing mode, which is current driven macro-MHD instability, because the $q$-profile has two $q=2$ resonant surfaces. ${ }^{2}$ The double tearing mode spreads between two resonant surfaces of $q=2$ at $r / a$

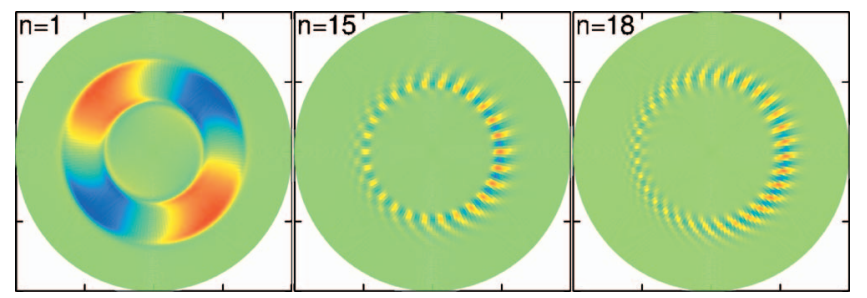

FIG. 2. (Color) The electric potential profile of eigenfunction on a poloidal section for instabilities: $n=1$ represents the double tearing mode; $n=15$ and 18 the kinetic ballooning modes.

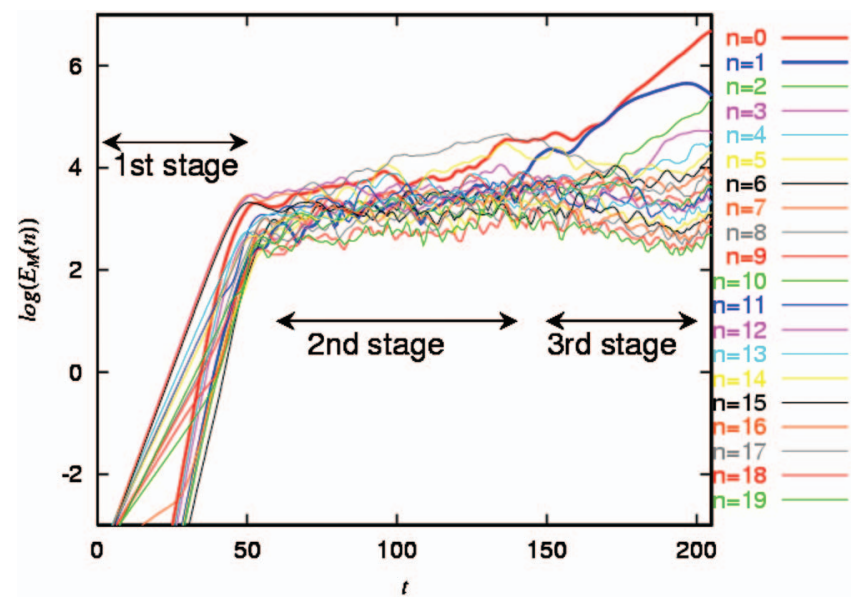

FIG. 3. (Color) Time evolution of the magnetic energy for each toroidal mode $n$, where the time is normalized by the ion thermal transit time. In the second stage a quasi-steady equilibrium is formed by a balance of turbulence with zonal flow. In the third stage a macro-MHD instability $(n=1)$ appears.

$=0.38$ and 0.69 as represented by the electric potential profile in Fig. 2 and grows faster than single tearing modes. The $n$ $=2$ and three macro-MHD modes are almost stable. The equilibrium is also unstable against the $6<n<22$ kinetic ballooning instabilities; ${ }^{17}$ i.e., microinstabilities. A ballooning structure of the microinstability appears in the bad curvature and positive shear region, as shown in Fig. 2. We start the nonlinear simulation at $t=0$, by taking the linear eigenfunctions of microinstabilities with small amplitudes as the initial condition. In order to obtain a quasi-steady equilibrium, we fix the background profiles of density and temperature during the simulation, while we do not fix the $q$-profile.

Nonlinear evolution consists of three subsequent stages. The first one is growth of microinstabilities within $0<t$ $<50$. The second one is a quasi-steady equilibrium formed by a balance between the turbulence and zonal flow within $60<t<140$. The third one is growth of a macro-MHD mode in $150<t$. Figure 3 shows time evolution of the magnetic energy for each toroidal mode number $n$. Figure 4 shows the

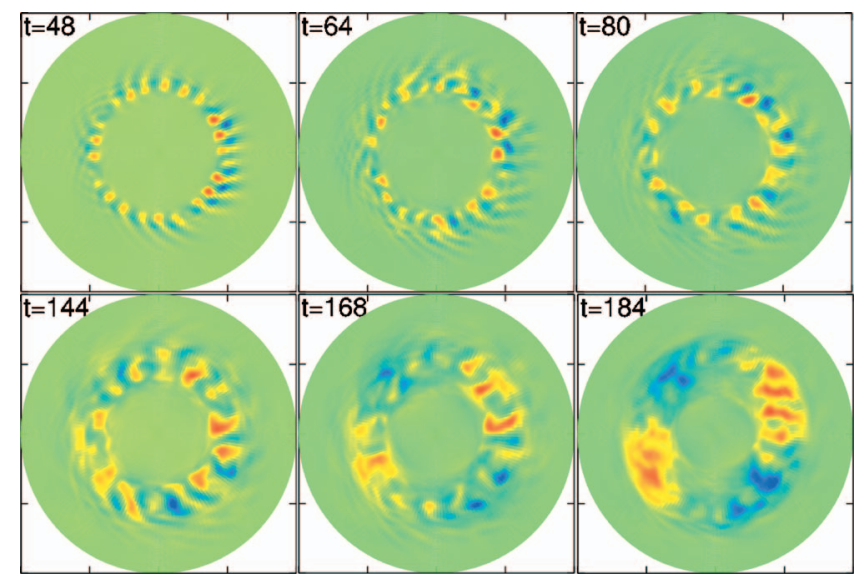

FIG. 4. (Color) Time evolution of the electric potential on the same poloidal section. 


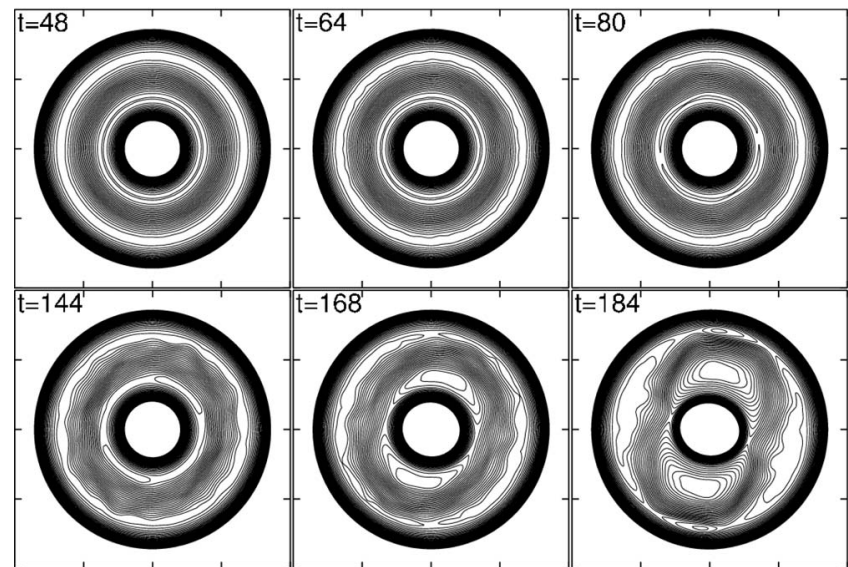

FIG. 5. Time evolution of the equicontour of helical flux of $m / n=2$ on the same poloidal section.

electric potential on a poloidal section at $t=48,64,80,144$, 168 , and 184 . Figure 5 shows the equicontour of helical flux of $m / n=2$ on a poloidal section.

In the first stage, i.e., $0<t<50$, the microinstabilities grow exponentially and nonlinear mode coupling among them rapidly drives the energy of both high and low wave numbers, as shown in Fig. 3. This is the energy transfer of microturbulence. The microturbulence also produces coherent structures including zonal flow with a $(m, n)=(0,0)$ structure and $n=1$ macroscale fluctuation through the energy transfer, as indicated by the traces with $n=0$ and $n=1$ in Fig. 3. The spatial profile of zonal flow is shown in Fig. 6(a). The zonal flow not only has $(m, n)=(0,0)$ structure but also has $(m, n)=(1,0)$ structure because of the geodesic acoustic mode. $^{18}$

In the second stage, i.e., $60<t<140$, we successfully obtain the quasi-steady equilibrium including turbulent fluctuations, zonal flow, and resonant surfaces of macro-MHD instability. The zonal flow twists the radial structure of the microinstability at $t=64$ in Fig. 4 and suppresses the growth of the instability, and then the system reaches a quasi-steady equilibrium in $t>60$, as shown in Fig. 3. The potential profile at $t=80$ presents a typical fluctuation of the turbulence in the quasi-steady equilibrium. Here we consider the $(m, n)$ $=(2,1)$ double tearing mode in this stage. There are no $\mathrm{m}$ $=2$ magnetic islands at two resonant surfaces in Fig. 5 within $60<t<140$, and the energy of $n=1$ mode fluctuates around
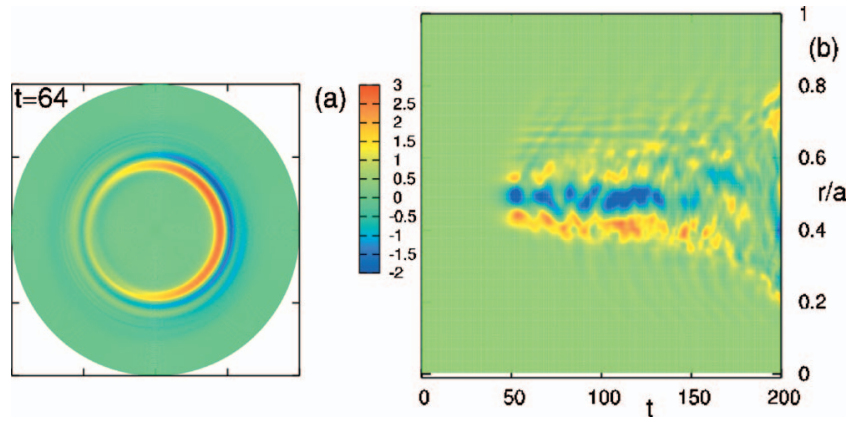

FIG. 6. (Color) (a) Spatial profile of zonal flow on a poloidal section. (b) Time evolution of zonal flow profile on a plane with $\theta=0$.

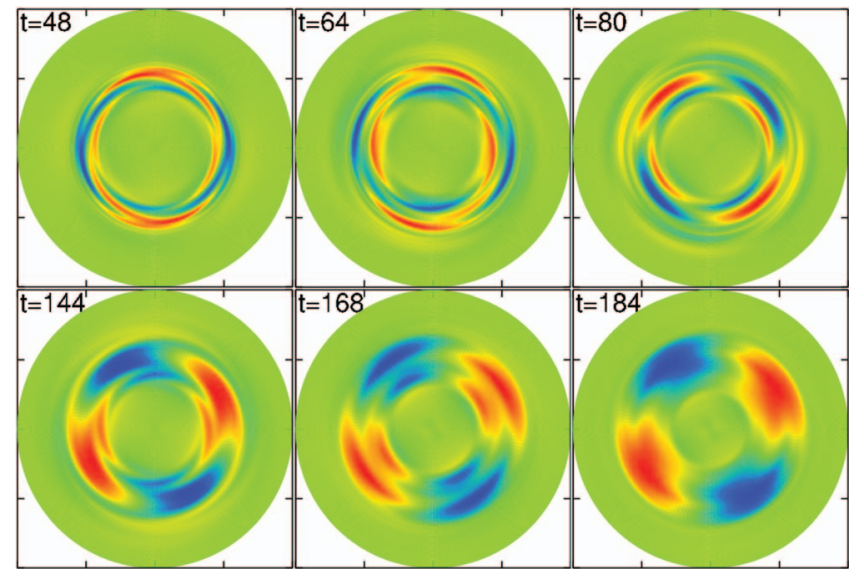

FIG. 7. (Color) Time evolution of the electric potential of $n=1$ mode on the same poloidal section.

the same amplitude as that of the turbulence indicated by high $n$ mode in Fig. 3. This is because the $(m, n)=(2,1)$ mode is a part of the turbulence; i.e., it is caused by the energy transfer of microturbulence. In addition, spatial distributions of the electric potential of $n=1$ mode at $t=64$ and 80 in Fig. 7 are strongly twisted by zonal flow and do not look like the eigenfunction of the double tearing mode in Fig. 2. The zonal flow has a stabilizing effect not only on the microturbulence but also on the tearing mode by twisting their radial structure.

In the third stage, i.e., $150<t$, the $(m, n)=(2,1)$ double tearing mode, i.e., the macro-MHD, appears. The energy of the $n=1$ mode grows from $t \approx 150$ and dominates at $t \approx 170$ in Fig. 3. The $m=2$ structure dominates the electric potential profile at $t=168$ and 184 in Fig. 4. In addition, two $m=2$ magnetic island-chains appear on two resonant surfaces at $t$ $=168$ and 184 in Fig. 5 .

Let us investigate the mechanism of macro-MHD instability in the third stage. The zonal flow has a stabilizing effect on the double tearing mode as explained above. Before appearance of a double tearing mode at $t=144$ there seems to be a randomly varying phase difference between the fluctuations on the inner and on the outer resonant surfaces, as shown in the equicontour of the helical flux in Fig. 5. This suggests that the reduction of the stabilizing effect of zonal flow can cause the macro-MHD instability. In fact, when the $(m, n)=(2,1)$ double tearing mode appears and dominates at $t \approx 170$ in Figs. 3 and 4 , the twisting by zonal flow vanishes and the spatial distributions at $t=168$ and 184 in Fig. 7 are similar to the eigenfunction of the double tearing mode in Fig. 2. The suppression of zonal flow is also observed at $t$ $>150$ in Fig. 6(b). The reduction of zonal flow can be caused by the torque of Maxwell stress acting on the poloidal flow. The stress is induced by the interaction between two magnetic island-chains, ${ }^{19}$ and it can weaken the shearing, thereby leading to the phase locking of two island-chains. The phase of X-point of inner islands corresponds to the phase of the O-point of outer islands, i.e., islands lock with a phase difference of $\pi$, at $t=168$ and 184 in Fig. 5 when the double tearing mode grows. At this time the plasma can effectively release the free energy of the equilibrium current 
density gradient. Once the magnetic islands grow they can produce stronger Maxwell stress. Hence, the mechanism of macro-MHD appearance is the positive feedback loop between zonal flow reduction and growth of magnetic islandchains.

Further, we consider effects of macro-MHD on the microturbulence. The macro-MHD, i.e., the tearing mode, can influence the microturbulence because the tearing mode breaks magnetic surfaces through global magnetic reconnection, as shown in the frame $t=168$ and 184 in Fig. 5. The radial distribution of turbulence at $t=184$ is broader than the distribution at $t=80$, as shown in the electric potential profiles in Fig. 4. This expansion is also observed in the evolution of the zonal flow profile at $t>160$ in Fig. 6(b). Thus, the reconnection spreads the microturbulence over the plasma.

We remark that the positive feedback loop producing macro-MHD occurs only when the equilibrium has resonant surfaces of MHD mode, otherwise the balanced state continues. That is confirmed by another simulation against initial equilibrium of $q_{\min }>2$. In this case, we have no growth of macro-MHD, i.e., double tearing mode, and have a steady state formed by a balance between the microturbulence and zonal flow.

In summary, we have found that the macro-MHD mode, $(m, n)=(2,1)$ double tearing mode, grows from the turbulent fluctuations in a quasi-steady equilibrium formed by a balance between the turbulence and zonal flow. Only after obtaining the quasi-equilibrium, which includes the microturbulence, zonal flow, and two resonant surfaces of $q=2$ are we able to obtain this simulation result of macro-MHD mode. The present multiscale simulation is more realistically comparable to the experimental observation of growing macro-MHD activity such as the one presented in Ref. 1 than earlier MHD simulations starting from linear macroinstability growth in a static equilibrium. This is because plasmas in experiments inherently include turbulent fluctuations and zonal flows and they apparently affect the growth of macroMHD mode through multiscale interactions.

Here we discuss how macro-MHD mode appears in the quasi-steady equilibrium. The mechanism of macro-MHD growth is the positive feedback loop between suppression of the zonal flow and growth of magnetic fluctuation. The suppression is caused by poloidal component of torque due to Maxwell stress through nonlinear interactions between magnetic fluctuations on two resonant surfaces, and then this torque causes phase locking between the fluctuations. The fluctuation grows as macro-MHD mode further when the zonal flow is suppressed, and then the fluctuations produce more strong torque. This positive feedback loop effectively releases the free energy of equilibrium current density gradi- ent and produces macro-MHD instability. The phase locking observed during the growth of the double tearing modes in our simulation is similar to the observation of $n=1$ macroMHD fluctuations in the reversed shear tokamak experiment. ${ }^{1}$ When the fluctuations are growing, the phases of fluctuations at two resonant surfaces of $q=3$ coincide, while phases differ before the growth.

The formation of quasi-steady equilibrium without macro-MHD instability can give a key to resolving the difficulty in tokamak plasma operations with $q_{\min }<2$. We do not understand the details of the quasi-equilibrium, i.e., the second stage, yet. The duration of the quasi-equilibrium can be affected by linear growth rate of macro-MHD instability and by the zonal flow damping due to collisions. Investigating this equilibrium in detail we can obtain a way to establish tokamak plasmas with $q_{\min }<2$ in the experiments.

\section{ACKNOWLEDGMENTS}

The authors would like to thank Dr. N. Miyato at JAEA for useful discussions. They also thank Professor S. Sudo for his support and Professor M. Skoric for his careful reading of this article.

One of the authors (A.I.) is supported by the Japanese Ministry of Education, Culture, Sports, Science, and Technology, Grant No. 18760642.

${ }^{1}$ S. Takeji, S. Tokuda, T. Fujita et al., Nucl. Fusion 42, 5 (2002).

${ }^{2}$ Y. Ishii, M. Azumi, and Y. Kishimoto, Phys. Rev. Lett. 89, 205002 (2002).

${ }^{3}$ A. Bierwage, S. Hamaguchi, M. Wakatani, S. Benkadda, and X. Leoncini, Phys. Rev. Lett. 94, 065001 (2005).

${ }^{4}$ G. T. A. Huysmans, T. C. Hender, N. C. Hawkes, and X. Litaudon, Phys. Rev. Lett. 87, 245002 (2001).

${ }^{5}$ P. H. Diamond, S.-I. Itoh, K. Itoh, and T. S. Hahm, Plasma Phys. Controlled Fusion 47, R35 (2005).

${ }^{6}$ W. Horton, Rev. Mod. Phys. 71, 735 (1999).

${ }^{7}$ K. Tanaka, C. Michael, A. L. Sanin et al., Nucl. Fusion 46, 110 (2006).

${ }^{8}$ D. Biskamp, Plasma Phys. Controlled Fusion 26, 311 (1984).

${ }^{9}$ P. H. Diamond, R. D. Hazeltine, Z. G. An, B. A. Carreras, and H. R. Hicks, Phys. Fluids 27, 1449 (1984).

${ }^{10}$ S.-I. Itoh, K. Itoh, and M. Yagi, Phys. Rev. Lett. 91, 045003 (2003).

${ }^{11}$ M. Yagi, S. Yoshida, S.-I. Itoh, H. Naitou, H. Nagahara, J.-N. Leboeuf, K. Itoh, T. Matsumoto, S. Tokuda, and M. Azumi, Nucl. Fusion 45, 900 (2005).

${ }^{12}$ C. J. McDevitt and P. H. Diamond, Phys. Plasmas 13, 032302 (2006).

${ }^{13}$ R. D. Hazeltine, M. Kotschenreuther, and P. J. Morrison, Phys. Fluids 28, 2466 (1985).

${ }^{14}$ B. Scott, Phys. Plasmas 7, 1845 (2000).

${ }^{15}$ X. Garbet, C. Bourdelle, G. T. Hoang, P. Maget, S. Benkadda, P. Beyer, C. Figarella, I. Voitsekovitch, O. Agullo, and N. Bian, Phys. Plasmas 8, 2793 (2001).

${ }^{16}$ N. Miyato, Y. Kishimoto, and J. Li, Phys. Plasmas 11, 5557 (2004).

${ }^{17}$ W. M. Tang, J. W. Connor, and R. J. Hastie, Nucl. Fusion 20, 1439 (1980).

${ }^{18}$ K. Hallatscheck and D. Biskamp, Phys. Rev. Lett. 86, 1223 (2001).

${ }^{19}$ R. Fitzpatrick and T. C. Hender, Phys. Fluids B 3, 644 (1991). 